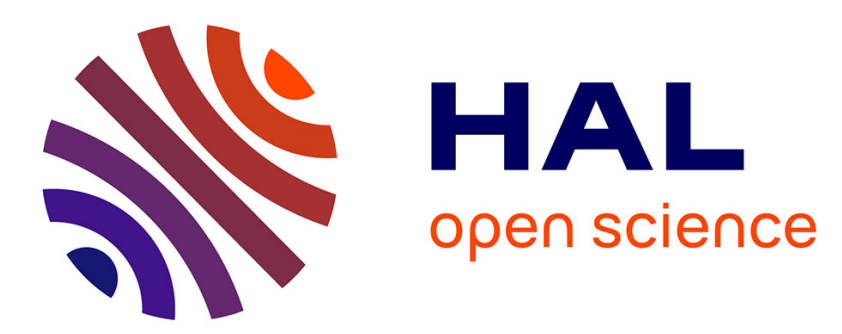

\title{
Laser-induced thermocapillary convective flows: A new approach for non-contact actuation at microscale at the fluid/gas interface
}

\author{
Ronald Terrazas, Aude Bolopion, Jean Beugnot, Pierre Lambert, Michaël
} Gauthier

\section{To cite this version:}

Ronald Terrazas, Aude Bolopion, Jean Beugnot, Pierre Lambert, Michaël Gauthier. Laser-induced thermocapillary convective flows: A new approach for non-contact actuation at microscale at the fluid/gas interface. IEEE/ASME Transactions on Mechatronics, 2017, 22 (2), pp.693 - 704. 10.1109/TMECH.2016.2639821 . hal-02300381

\section{HAL Id: hal-02300381 https://hal.science/hal-02300381}

Submitted on 29 Sep 2019

HAL is a multi-disciplinary open access archive for the deposit and dissemination of scientific research documents, whether they are published or not. The documents may come from teaching and research institutions in France or abroad, or from public or private research centers.
L'archive ouverte pluridisciplinaire $\mathbf{H A L}$, est destinée au dépôt et à la diffusion de documents scientifiques de niveau recherche, publiés ou non, émanant des établissements d'enseignement et de recherche français ou étrangers, des laboratoires publics ou privés. 


\title{
Laser-induced thermocapillary convective flows: A new approach for non-contact actuation at microscale at the fluid/gas interface
}

\author{
Ronald Terrazas Mallea ${ }^{1,2}$, Aude Bolopion ${ }^{2}$, Jean-Charles Beugnot ${ }^{2}$, Pierre Lambert ${ }^{1}$, Michael Gauthier ${ }^{2}$
}

\begin{abstract}
This work proposes a new actuation principle for non-contact actuation. Thermocapillary convection is a promising principle to manipulate particles at the fluid/gas interface. Compared to approaches based on natural and Marangoni convections, our approach uses thermocapillary convection generated by a laser heating the fluid from the top, and not from the bottom. This has several advantages, being the most relevant that it does not depend on an hydrodynamic instability to onset the flow motion. Laser heating creates large, localized thermal gradients that make the flow velocity fast and localized. Simulations show that flow velocities up to $8.5 \mathrm{~mm} / \mathrm{s}$ can be obtained using as little power as $38 \mathrm{~mW}$ with a temperature increase as little as $4^{\circ} \mathrm{C}$. As a proof of concept, steel spherical particles of $500 \mu \mathrm{m}$ diameter are successfully displaced using this principle, which attain a mean maximal speed up to $4 \mathrm{~mm} / \mathrm{s}$. Also, $1000 \mu \mathrm{m}$ diameter steel spherical particles are displaced along a given trajectory using a manual control. These first results demonstrate the high potential of this new approach based on thermocapillary convection for controlled non-contact actuation at high speeds at microscale.
\end{abstract}

\section{INTRODUCTION}

Non-contact micromanipulation has received a lot of interest in recent years. Different from the contact manipulation techniques that require the actuator to stay in touch with the target object, non-contact micromanipulation uses force fields remotely applied to manipulate the object. Several actuation principles are available that present specific advantages and drawbacks in terms of applied force, linearity of the force fields, characteristic of the objects that can be manipulated, etc.

Magnetic actuation is one of these actuation principle [1]. Magnetic fields produced either by permanent magnets or by electromagnets are used to apply a force and a torque to magnetic objects. The objects can be controlled either in dry environments [2] [3], inside liquids [4] or at the interface between two fluids [5]. Depending on their magnetic susceptibility the objects are either attracted toward the zones of high magnetic field gradient [6], or they are repelled from them [7]. Even though there exist some techniques to address independently several magnetic tools, it remains a major bottleneck to control more than a few of them simultaneously [8].

Electrical fields are also a widely used solution to control micrometer size objects. Dielectrophoresis consists in applying a variable electrical field, which produces a force on any dielectric object. This force depends on the object properties, the medium properties, and the frequency and amplitude of

${ }^{1}$ Bio-, Electro- And Mechanical Systems department (BEAMS), Ecole Polytechnique de Bruxelles, Universite Libre de Bruxelles, 1050 Bruxelles, Belgium. rterraza@ulb.ac.be

2 FEMTO-ST Institute, AS2M and Optics departments, Univ. Bourgogne Franche-Comté, Univ. de Franche-Comté/CNRS/ENSMM, 24 rue Savary, F25000 Besançon, France aude.bolopion@femto-st. fr the electrical field [9], [10]. It is commonly used for biological applications [11], [12]. Precise trajectory control can also be performed. As an example, controlled trajectories of artificial glass beads of $40 \mu \mathrm{m}$ placed on a petri dish using visual based closed-loop control have been performed [13]. Again, all the dielectric objects will be affected by the dielectrophoretic force.

Optical tweezers use a laser beam to control micro-objects. The physical principle is based on the pressure radiation of the light. The objects should be transparent, and their refractive index should be higher than the one of the environment. The objects are attracted toward the region of the highest light intensity. By moving the laser beam it is possible to control the position of the objects [14]. This technique can be applied to objects whose size ranges from tens of nanometers to a few micrometers [15]. Optical tweezers benefit from a high selectivity since only the object placed on a close vicinity to the laser is attracted toward the center of the beam [16]. This approach has been used for cell manipulation [17] and pairing of cells from different groups [18].

Manipulation via actuated flows uses the flow motion to move the desired object. Different mechanisms can be used to onset the flow motion like: electroosmosis, electrohydrodynamics, micromechanical, thermocapillary pumping [19], among others. This technique has been already used for cell manipulation [20]-[22]. In [23], is shown that hollow glass microspheres which are heavier and larger than cells can also be manipulated. This is possible because the drag force scales with the size of the component.

In this paper, a novel approach is proposed to manipulate microscale particles using non-contact actuation based on actuated flows. The proposed technique is based on thermocapillary convection generated by laser heating from the top of the sample to control the position of particle lying at the interface. This configuration offers several advantages that will be detailed in the paper. One of them is the use of thermocapillary convection, which is generated by a surface tension stress being this a surface effect. This is advantageous because it is known that at the microscale, the surface effects are dominant over the volumetric ones. In addition, it can be generated using small temperature increases and does not depend on an instability mechanism to generate flow. In section II, the physical principle is described together with a comparison against other manipulation techniques that also use surface tension stresses. In section III, the thermocapillary flow is simulated and the results are used to explain the specific characteristics of the current proposal. Section IV presents a proof of concept where a $500 \mu \mathrm{m}$ steel spherical particle is displaced using the current proposal, and allows to compare it 
against others techniques. It also shows that a $1000 \mu \mathrm{m}$ steel spherical particle can be moved along a trajectory using a manual control. The conclusions of this work are presented in section V.

\section{PHYSICAL PRINCIPLE}

\section{A. Surface tension}

Surface tension $(\sigma)$ can be defined as the required energy to increase the area of an interface by one unit [24]. It is known that surface tension depends almost linearly on temperature when the fluid temperature is below a critical value $(653 \mathrm{~K}$ for water [25]), according to Eötvös equation for pure liquids [26]. For most liquids, $\sigma$ decreases with temperature. Let us consider a surface element $L \cdot d x$, where $L$ is the length of the surface element in the y-direction. Let us consider this element on an interface that has a thermal gradient such that $T_{1}>T_{2}$, where $T_{1}$ and $T_{2}$ are the temperatures at edges 1 and 2, respectively. Because liquids' surface tension decreases with temperature, $\sigma_{1}$ is lower than $\sigma_{2}$ and therefore, there is a net force $F_{2}-F_{1}$ pulling the hot edge towards the colder one (Fig. 1). This resulting surface tension force creates a shear stress $\tau^{(\sigma)}$ in the surface element $L \cdot d x$ in the X-direction. Using the chain rule, this stress can be expressed as:

$$
\tau^{(\sigma)}=\frac{\partial F}{L \cdot \partial x}=\frac{\left(\sigma_{2}-\sigma_{1}\right) L}{L \cdot \partial x}=\frac{\partial \sigma}{\partial x}=\frac{\partial \sigma}{\partial T} \frac{\partial T}{\partial x}
$$

On a free liquid surface, the surface tension stress $\tau^{(\sigma)}$ is in equilibrium with the viscous stress $\tau^{(\nu)}$. This equilibrium is expressed as:

$$
\tau^{(\sigma)}+\tau^{(\nu)} \equiv \frac{\partial \sigma}{\partial T} \frac{\partial T}{d x}-\mu \frac{\partial v_{x}}{\partial z}=0
$$

where:

$\mu$ is the dynamic viscosity $\left[\mathrm{N} \mathrm{s}^{-1} \mathrm{~m}^{-2}\right]$

$v_{x}$ is the fluid velocity in the $\mathrm{x}$-direction $[\mathrm{m} / \mathrm{s}]$

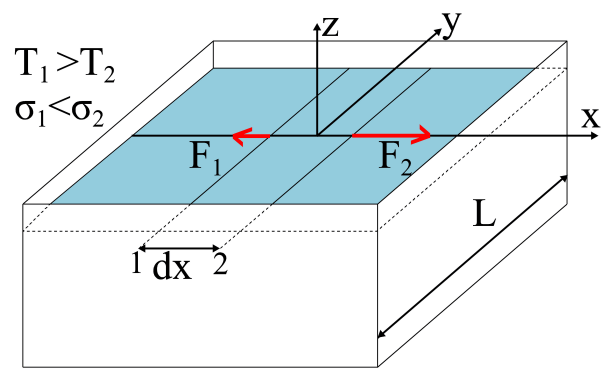

Fig. 1: Force representation at the fluid/gas interface on an elementary surface element $L \cdot d x$ between edges 1 and 2 . When $T_{1}>T_{2}$, the surface tension at edge 1 is lower than at edge $2 \sigma_{1}<\sigma_{2}$, therefore $F_{1}<F_{2}$, and so there is a net force towards the cold point 2 .

The equation (2) becomes a boundary condition in the Navier-Stokes' equations and is responsible for the onset of the fluid motion. The value of $\frac{\partial \sigma}{\partial T}$ is a negative constant, and so, the strength of the surface tension stress depends on the value of the temperature gradient $\frac{\partial T}{\partial x}$. So, the larger the generated temperature gradient, the stronger the generated flow will be.

\section{B. Thermal-driven convective flows}

Flows generated by thermal induced surface tension gradients enter under the category of thermal-driven convective flows. To position the contribution of this work, let us consider the three types of thermal driven convections: natural, Marangoni and thermocapillary.

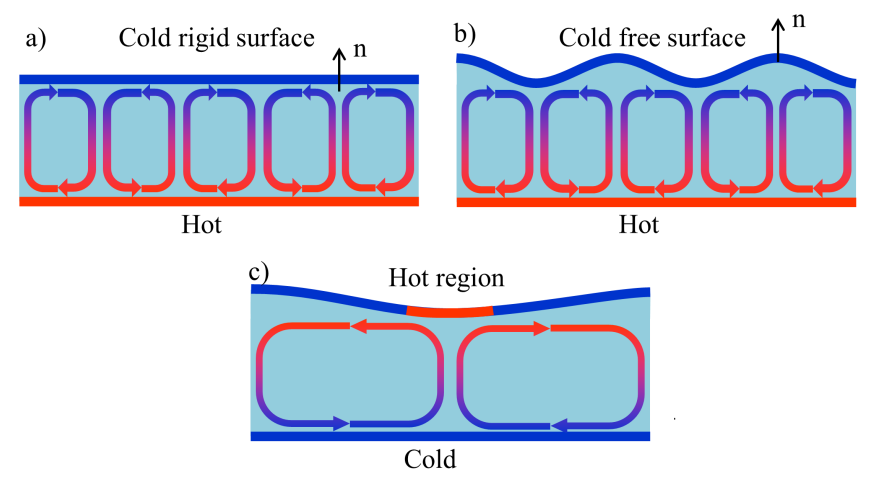

Fig. 2: Representation of the different types of thermal-driven convections: a) natural, b) Marangoni and c) thermocapillary.

Natural convection (Fig. 2a) occurs due to a density gradient along the axis $\mathbf{n}$, normal to the fluid surface, produced by a temperature gradient when the fluid is heated from below. Its driving principle is the Rayleigh-Bénard instability [27]. The occurrence of the instability is assessed by the Rayleigh number, which has to surpass the critical value of $R a_{\text {crit }}=$ 1708 [23], [27].

Marangoni convection (Fig. 2b) is a second type of convection principle which occurs due to a surface tension gradient on the fluid free upper surface, produced by a temperature gradient on the interface when the fluid is heated from the bottom. Its driving principle is the Marangoni-Bénard instability [27], [28]. The occurrence of the instability is assessed by the Marangoni number, which has to surpass the critical value of $M a_{\text {crit }}=81$ [23], [27].

Thermocapillary convection (Fig. 2c) is a third type of convection principle which occurs due to a surface tension gradient parallel to the fluid free upper surface, produced by a temperature gradient on the interface when the fluid is heated from the top. Although the physical driving principle is the same as for the Marangoni convection, the physical setting is different. Because the heat is coming from the top, the temperature gradient at the interface is directly generated by the heat source. Consequently, any temperature gradient will generate thermocapillary convection, so there is no critical or threshold temperature gradient value [28]. Moreover the generation of thermocapillary convection does not generate natural convection as the heat comes from the top.

\section{State of the art on manipulation using Marangoni and thermocapillary convections}

At microscale, surface effects are dominant over volume effects. This means that stronger forces can be obtained using surface tension forces than using buoyant forces. Therefore, 
the surface tension driven mechanisms: Marangoni and thermocapillary convection are preferred over the natural convection mechanism.

Marangoni and thermocapillary convections have been used as actuation principles for micromanipulation. In [29] and [23], the authors used a laser to heat the bottom of a container filled with water to generate Natural and Marangoni convections on a closed-loop system. Glass beads particles of sizes up to $92 \mu \mathrm{m}$ are manipulated with experimental speeds up to $5.5 \mathrm{~mm} / \mathrm{s}$ and an estimated actuation force of $40 \mathrm{pN}$. In [30], the authors used resistors to heat a water/air interface, generating thermocapillary flow which was used for the sedimentation of weed pollen Kochia scoparia particles of diameter of $25 \mu \mathrm{m}$. In [31], the authors heated the bottom of a substrate to generate Marangoni convection for the sorting of hollows glass cenospheres of sizes between 50-200 $\mu \mathrm{m}$. A slightly different concept was presented in [32], where the authors injected air bubbles on a closed container filled with silicone oil and generated thermocapillary convection by heating the bubble/fluid interface. Depending if the particle was sunk or floating in the bulk, it was affected by the fluid going inwards or outwards the bubble respectively. Using this concept, they manipulated $120 \mu \mathrm{m}$ diameter glass beads with an estimated force of $1.6 \mathrm{nN}$ when pushing a floating particle and $0.6 \mathrm{nN}$ when pulling a sunk particle inwards the bubble. In [33], instead of inserting a bubble, the authors used a focalized laser beam to heat an absorbent substrate strongly enough to generate a small air bubble at the bottom of the fluid bulk. By heating the fluid/bubble interface, they generate thermocapillary convection. They used this concept to manipulate $20 \mu \mathrm{m}$ diameter polystyrene particles. The reported measured speeds were of $200 \mu \mathrm{m} / \mathrm{s}$ when pulling sunk particles and $100 \mu \mathrm{m} / \mathrm{s}$ when pushing floating particles in the fluid bulk.

Between Marangoni and thermocapillary convections, the second one presents several advantages. Because the temperature gradient on the surface is generated directly by the heat source, there is no temperature threshold. Moreover, it does not depend on the fluid layer thickness, it is more energy efficient than the Marangoni convection and a steady flow can be obtained if the temperature gradient is not too large [28]. For these reasons, thermocapillary convection is selected over Marangoni convection for non-contact manipulation at microscale.

\section{PHYSICAL MODELING}

As seen in section II-A, two main physics are involved in the generation of thermocapillary convective flow: heat transfer modeled by the Heat equation and fluid dynamics modeled by the Navier-Stokes' equations, both linked by the stress equilibrium condition (2). In this section, the system to be considered is presented together with a recapitulation of the different phenomena involved. The mathematical equations defining the system are analyzed in order determine the dominant terms and its effect in the temperature and the flow velocity.

\section{A. System description}

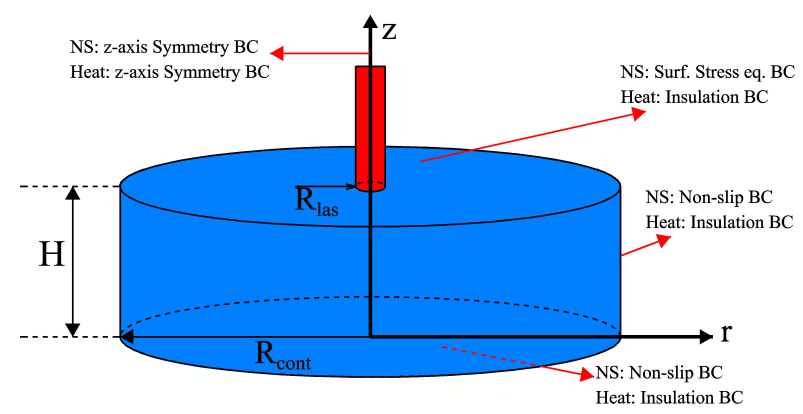

Fig. 3: System geometrical parameters representation. A laser heats the fluid as defined by the heat equation. This generates a stress at the water/air interface which generates the flow motion, modeled by the Navier-Stokes' (NS) equations. The boundary conditions (BC) used in the heat and Navier-Stokes' equations are shown.

Figure 3 shows a schematic representation of the system. A container of radius $R_{\text {cont }}$ contains a water layer of height $H$ initially at state of repose $(\mathbf{v}=\mathbf{0})$ and at ambient temperature $T_{0}$ at time $t=0 \mathrm{~s}$. After that, for the reasons which are explained in section III-B, a collimated incident laser beam with waist radius of $R_{\text {las }}$ and power $P_{\text {las }}$ coming from the top, reaches the surface of the water at point $(r=0, z=H)$. According to Beer-Lambert's law, part of this energy will be absorbed by the liquid as it passes through which is characterized by the absorption coefficient $\alpha$. This coefficient is specific for a given laser wavelength and medium. This absorbed energy will be converted to heat and will be propagated in the fluid. This phenomenon is modeled by the heat equation which defines the temperature field in the entire fluid. Then, according to equation (2), the temperature gradient at the interface will generate a surface tension stress at the interface, which will propagate in the fluid. A stress is a form of flow momentum, and the momentum transfer is defined by the Navier-Stokes' equations, which define the velocity field across the entire fluid.

\section{B. Laser Heating}

Among the different possibilities to heat the fluid/gas interface, laser heating has the advantage of creating large localized temperature gradients [34], which boosts the thermocapillary convective flow. In order to corroborate this, let us analyze the equation representing the laser heating.

The laser beam used in the system is collimated, meaning that its radius does not change significantly. Also, the water absorption is significant $(\alpha H \gg 1)$. Taking these two points into consideration, the suitable mathematical expression for the laser heating term $Q_{\text {laser }}$ is [34]:

$$
Q_{\text {laser }}=\frac{2 P_{\text {las }} \alpha}{\pi R_{\text {las }}^{2}} \exp \left(\frac{-2 r^{2}}{R_{\text {las }}^{2}}-\alpha(H-z)\right)
$$

where:

$H$ liquid layer height [m]

$P_{\text {las }}$ laser power [W] 
$r$ radial-axis coordinate $[\mathrm{m}]$

$R_{\text {las }}$ laser beam collimated waist radius [m]

$z$ axial coordinate from liquid surface $[\mathrm{m}]$

$\alpha$ absorption coefficient of the liquid $\left[\mathrm{m}^{-1}\right]$

Let us consider the heat source at the interface $(z=\mathrm{H})$, where the remaining term in the exponential term $\frac{-2 r^{2}}{R_{\text {laser }}}$, defines an exponential decrease in the radial direction which is even steeper due to the quadratic terms. So at $r=R_{\text {laser }}$ $86.47 \%$ of the laser power is already absorbed and at $r=$ $2 R_{\text {laser }} 99.97 \%$ is absorbed. This significant decay allows the generation of large temperature gradients at the interface, which boost the thermocapillary flow.

Another advantage is that the heated area can be tuned by changing the waist radius of the laser. So with small beam radius, small regions can be heated, meaning that the force will be applied on determined regions of the workspace. This is an advantage compared to other methods in which the force is applied in the entire workspace (e.g. magnetic manipulation).

\section{Mathematical modeling}

The problem of modeling the convective flow has already been tackled by different authors. Models for the Marangoni convective flow due to continuous laser heating were proposed in [23], [35], models for the thermocapillary convective flow due to continuous laser heating were proposed in [34], [36] and for pulsed laser heating in [37]. These models were used as a base to propose a suitable model for the current system.

The system can be considered symmetrical around the Zaxis (incident laser beam axis) for the temperature field, and consequently, for the velocity field. So, the 3D problem can be reduced to a 2D problem in cylindrical coordinates. Thermophysical properties are considered constant, except for the surface tension which depends on the temperature and the fluid density. The last one is modeled using the Boussinesq approximation [38] in the Navier-Stokes' equations in order to consider the buoyancy force.

Taking into account those considerations, the Navier-Stokes' equations can be written as:

$$
\rho\left(\frac{\partial \mathbf{v}}{\partial t}-(\mathbf{v} \boldsymbol{\nabla}) \mathbf{v}\right)=\mu \boldsymbol{\nabla}^{2} \mathbf{v}-\boldsymbol{\nabla} p+\mathbf{g} \beta\left(T-T_{0}\right)
$$

The conservation of mass equation for an incompressible fluid is:

$$
\nabla \mathbf{v} \equiv \frac{\partial v_{r}}{\partial r}+\frac{v_{r}}{r}+\frac{\partial v_{z}}{\partial z}=0
$$

The heat equation for the system may be written as:

$$
\rho C_{p}\left(\frac{\partial T}{\partial t}-(\mathbf{v} \nabla) T\right)=k \boldsymbol{\nabla}^{2} T+Q_{\text {laser }}
$$

Subjected to the following boundary conditions:

$$
\begin{array}{rlrl}
\frac{\partial v_{r}}{\partial z} & =\frac{1}{\mu} \frac{\partial \sigma}{\partial T} \frac{\partial T}{\partial r} & & z=H \\
\mathbf{v} & =0 & & z=0 \\
\mathbf{v} & =0 & & r=R_{\text {cont }} \\
\frac{\partial v_{r}}{\partial r} & =0 & r & =0 \\
p & =0 & & \left(r=R_{\text {cont }}, z=H\right) \\
\mathbf{v} & =0 & & t=0
\end{array}
$$

$$
\begin{array}{rlrl}
\frac{\partial T}{\partial z}=0 & z & =H \\
\frac{\partial T}{\partial z}=0 & & z=0 \\
\frac{\partial T}{\partial r}=0 & r & =R_{\text {cont }} \\
\frac{\partial T}{\partial r}=0 & r & =0 \\
T & =T_{0} & t & =0
\end{array}
$$

where:

$C_{p}$ specific heat $\left[\mathrm{J} \mathrm{kg}^{-1} \mathrm{~K}^{-1}\right]$

g gravity acceleration vector $\left[\mathrm{m} / \mathrm{s}^{2}\right]$

$H$ liquid layer height $[\mathrm{m}]$

$k$ thermal conductivity $[\mathrm{W} /(\mathrm{m} \mathrm{K})]$

$p$ pressure $\left[\mathrm{N} / \mathrm{m}^{2}\right]$

$R_{\text {cont }}$ radius of the liquid container [m]

$r$ radial-axis coordinate $[\mathrm{m}]$

$T$ temperature $[\mathrm{K}]$, where subscript 0 denotes the ambient temperature

$t$ time [s]

$\mathbf{v}$ flow velocity vector $[\mathrm{m} / \mathrm{s}]$, where subscripts $r$ and $z$ denote the radial and axial direction respectively

$z$ axial coordinate from liquid surface $[\mathrm{m}]$

$\beta$ is the coefficient of thermal expansion, $\beta=\frac{1}{\rho} \frac{d \rho}{d T}\left[\mathrm{~K}^{-1}\right]$

$\mu$ is the dynamic viscosity $\left[\mathrm{N} \mathrm{s}^{-1} \mathrm{~m}^{-2}\right]$

$\rho$ is the density of the fluid $\left[\mathrm{kg} / \mathrm{m}^{3}\right]$

$\sigma$ surface tension of the fluid $[\mathrm{N} / \mathrm{m}]$

Equations (7) and (8) denote the boundary conditions (BC), for the Navier-Stokes' and heat equations respectively. For the Navier-Stokes' equations, a non-slip wall BC is assumed at the bottom (7b) and at the edge of the container (7c), an axisymmetric $\mathrm{BC}$ around the $\mathrm{Z}$-axis (7d), the stress equilibrium $\mathrm{BC}$ at the interface (7a), and the fluid is considered still at the beginning (7f).

In the heat equation it is considered the heat transfer between the air and the water is negligible, so an insulation BC is assumed for the interface (8a). An insulation BC is used at the bottom (8b) and at the edge of the container (8c) because the container is made of plastic which is a good insulator. An axi-symmetric $\mathrm{BC}$ is used around the $\mathrm{Z}$-axis (8d) and the fluid is considered at ambient temperature at the beginning (8e).

\section{Analysis of the heat and momentum transfer}

This section proposes a discussion on the physics involved in the system and how they affect the resulting thermocapillary flow.

Heat and momentum transfer are present in the system. It is important to remember that there are 2 main transfer mechanisms: diffusion and convection. Diffusion is the transfer of a property (momentum or heat) due to the contact of fluid molecules regardless of the fluid movement. On the other hand, convection is the transfer of a property due to the movement of fluid. The heat equation (6) models the heat transfer on a system, represented by the temperature. The diffusive transfer mechanism is represented by the term $k \nabla^{2} T$ and the convective transfer mechanism is given by the term 
$\rho C_{p}(\mathbf{v} \nabla) T$. The ratio between the convective and diffusive mechanisms is given by the thermal Peclet number, which is defined as $P e_{t h}=\frac{\rho C_{p} U L}{k}$, where $L$ is a characteristic distance, the height of the liquid layer in this case and $U$ is a characteristic velocity. As it can be seen in table I, the Peclet number attains a maximum of 460 at the interface near the laser spot, and values below 40 elsewhere. In consequence, the convective transfer mechanism is dominant in the system meaning that the flow velocity field will have a significant effect in the temperature field.

TABLE I: Comparison dimensionless numbers

\begin{tabular}{|c|c|c|c|c|}
\hline$r[\mathrm{~mm}]$ & $z[\mathrm{~mm}]$ & $V_{r} \mathrm{~mm} / \mathrm{s}$ & $P e_{\text {th }}$ & $R e$ \\
\hline 0.83 & 7.5 & 8.7 & 460 & 65 \\
\hline$\approx 25$ & 7.5 & 0.5 & 26 & 3.7 \\
\hline 1 & 6.4 & -0.62 & 36 & 5.2 \\
\hline
\end{tabular}

On the other hand, the Navier-Stokes' equations (4) model the momentum transfer, represented by the velocity. The diffusive transfer mechanism is given by the term $\mu \nabla^{2} \mathbf{v}$ and the convective transfer mechanism is given by the term $\rho\left(\begin{array}{ll}\mathbf{v} & \boldsymbol{\nabla}\end{array}\right) \mathbf{v}$. In this case, the ratio between the two is given by the Reynolds number defined as $R e=\frac{\rho U L}{\mu}$. As it can be seen in table I, the maximum attained Reynolds number is obtained at the interface near the laser spot with a value of 65 , and values below 6 elsewhere. This means that the convective transfer mechanism is the dominant one. However, because the values are below 2200, the flow is considered to be laminar and turbulences are not present.

Because the convective mechanism dominates the momentum and heat transfer, the behavior of the flow velocity will have a significant effect in the system. The dynamics imposed by the solution of the Navier-Stokes' equations will have a significant effect in the dynamics of the heat equation because of the coupling between both phenomena through the $\mathrm{BC}$ at interface.

\section{Finite ELEMENT Simulations}

The heat and Navier-Stokes' equations model the temperature and fluid velocity in the entire fluid. The flow motion will push a particle lying at the water/air interface due to drag force. In the case of a spherical particle, this force can be estimated using the Stokes' law, once the entire velocity field is known. Therefore, simulations are carried on using the experimental conditions to determine fluid velocity which will be then used to estimate the particle velocity in section V-B.

Simulations are performed in COMSOL 5.0 software, using the "Laminar Flow" module for fluid dynamics physics. Since the Reynolds number obtained is below 2200, the flow is considered to be laminar and so this module can be used with confidence in the results. The "Heat Transfer in Fluids" module is used for the thermal physics. A 2D axisymmetric space is considered. The geometrical and solver parameters are shown in table II.
TABLE II: System and solver parameters for COMSOL

\begin{tabular}{|l|c|}
\hline \multicolumn{2}{|c|}{ System parameters } \\
\hline$P_{\text {las }}[\mathrm{mW}]$ & 37 \\
\hline$\alpha\left[\mathrm{m}^{-1}\right]$ & 3280 \\
\hline$L_{\alpha}=\alpha^{-1}[\mu \mathrm{m}]$ & $\approx 305$ \\
\hline$k\left[\mathrm{~W} \mathrm{~m}^{-1} \mathrm{~K}^{-1}\right]$ & 0.607 \\
\hline$\mu\left[\mathrm{N} \mathrm{s}^{-1} \mathrm{~m}^{-2}\right]$ & 0.001002 \\
\hline$\rho\left[\mathrm{Kg} / \mathrm{m}^{3}\right]$ & 997 \\
\hline$\frac{\partial \sigma}{\partial T}\left[\mathrm{~N} \mathrm{~m}^{-1} \mathrm{~K}^{-1}\right]$ & 0.00001533 \\
\hline$H[\mathrm{~m}]$ & 0.0075 \\
\hline$R_{\text {las }}[\mathrm{m}]$ & $625 \times 10^{-6}$ \\
\hline$T_{0}[\mathrm{~K}]$ & 293.15 \\
\hline$C_{p}\left[\mathrm{~J} \mathrm{~kg}{ }^{-1} \mathrm{~K}^{-1}\right]$ & 4181 \\
\hline \multicolumn{2}{|c|}{ COMSOL solver parameters } \\
\hline Solver & Generalized alpha \\
\hline Rel. Tolerance & $10^{-6}$ \\
\hline Abs. Tolerance & $10^{-5}$ \\
\hline Init. Step time & $10^{-6} \mathrm{~s}$ \\
\hline Max. Time step & $0.05 \mathrm{~s}$ \\
\hline
\end{tabular}

\section{A. Temperature and radial flow velocity at the interface}

Let us go deeper in the initial description of the system presented at the beginning of section III. At time $t_{0}$, the water is at repose state and at ambient temperature. At time $t_{0^{+}}$, the laser starts heating the water setting an initial temperature gradient, which generates a surface tension stress at the water/air interface. Due to the stress equilibrium condition at the interface (7a), the surface tension stress generates a flow motion. However, as the convective transfer mechanism dominates the heat transfer, this flow is also transferring heat to the peripheries of the heated region. This creates a heat-momentum propagation mechanism, on which the flow motion transfers heat to the peripheries of the heated region, expanding the range of the temperature gradient, which at the same time expands the range of the flow motion allowing to transfer the heat even further.

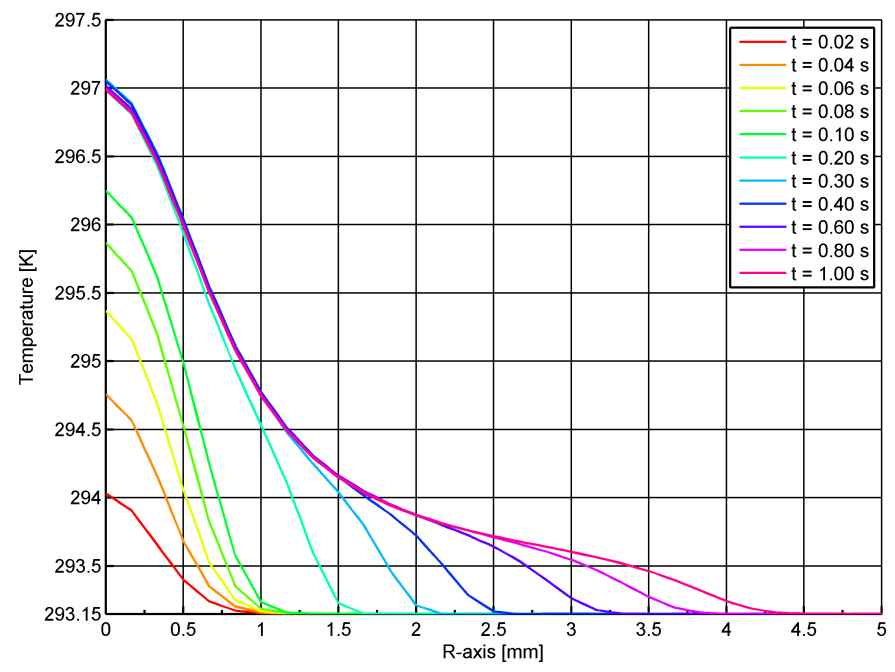

Fig. 4: Temperature temporal evolution at the water/air interface $(z=H)$ between $0-1 \mathrm{~s}$.

This can be appreciated on figure 4, which shows the temperature at the interface in the first time instants up to 1 
second in function of the radial coordinates. At the initial time instants, the temperature begins to rise but the temperature gradient is not large, and so the flow motion is weak and does not contribute much to propagate the heat. However, after $t=$ $0.2 \mathrm{~s}$, the temperature gradient begins to generate a significant flow motion that propagates the heated region at each time instant, expanding also the range of the flow motion.

The same explanation is also valid for larger periods of time as it can be seen in figures $5 \mathrm{a}$ and $5 \mathrm{~b}$, which show the temperature and radial velocity at the interface in function of the radial coordinates. The temperature and radial velocity field affect a similar area at each time instant, and propagate similarly as time passes. It can be noted the existence of an inflection point on both the temperature and the radial flow velocity fields at the periphery of the heated region. This is due to the coupling between the heat transfer and the flow motion. Heat cannot propagate further because it requires the flow motion to cover a larger distance and, on the other hand, the flow motion cannot cover a larger distance without a bigger heated region. Therefore, both phenomena limit each other and that is why both propagate almost at the same velocity.

As time passes, it becomes harder to transfer heat to the periphery of the heated region because of the localized nature of the laser heating. The temperature gradient generated at the edge of the expanding heated region becomes weaker, which also generate weak flow motions at the periphery which slows down the overall propagation process. This can be noted in both figures $5 \mathrm{a}$ and $5 \mathrm{~b}$, where it took around 10 $\mathrm{s}$ to propagate both heat and momentum up to a distance of $\approx 13 \mathrm{~mm}$, while it took around $60 \mathrm{~s}$ to propagate them up to a distance of $25 \mathrm{~mm}$ (12 $\mathrm{mm}$ more).

Having analyzed the common characteristics of both plots, let us now consider the specific characteristics of each plot. Regarding the temperature against the radial coordinates shown in Fig. 5a, it can be seen that there is a steep decay in the temperature near the origin. This was expected due to the localized nature of the laser heating. Another point to notice is the low temperature increase which is approximately $4{ }^{\circ} \mathrm{K}$ at the center of the laser spot (origin), however because of the steep decrease, at $r=1 \mathrm{~mm}$ the temperature increase is only $1.55^{\circ} \mathrm{K}$ and at $r=3 \mathrm{~mm}$, only $1^{\circ} \mathrm{K}$. This is interesting because temperature sensible particles could be manipulated, and also means that particles can be manipulated without a significant change on its temperature dependent properties, which may be the case of electrical conductivity for example.

Let us now consider the radial flow velocity against the radial coordinates shown in Fig. 5b. An overshoot in the flow velocity can be noticed at $t=0.2 \mathrm{~s}$. In fact the temperature also presented an overshoot but it was very small. This occurs because the coupling between the heat and NavierStokes equations define a second-order system behavior in the temperature and the flow velocity. Another point to notice is that the maximum radial flow velocity does not occur at the origin but a certain distance away. This sets a clear difference in relation to the optical tweezers that need to point the particle to manipulate with the laser. In the system proposed, the laser would need to be pointed at a certain distance from the particle. Another interesting result is that despite the low temperature increase, radial flow velocities up to $8.5 \mathrm{~mm} / \mathrm{s}$ are obtained. This is already very promising towards the particle manipulation compared to the particle velocities up to $5.5 \mathrm{~mm} / \mathrm{s}$ reported in [23].

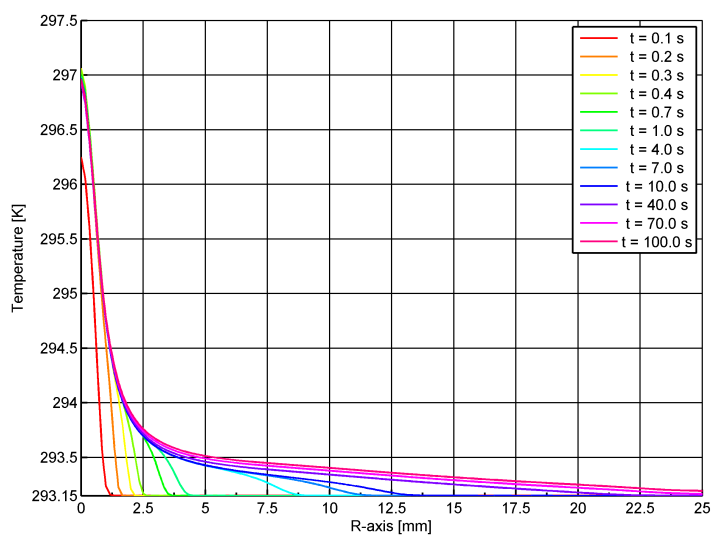

(a) Surface temperature

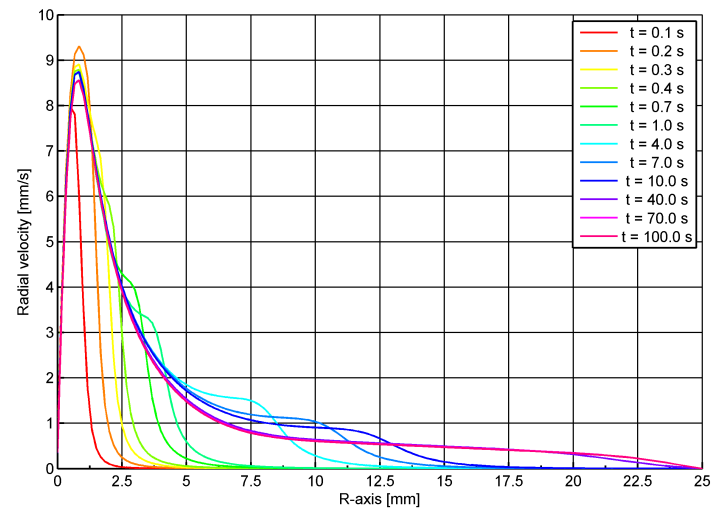

(b) Surface radial velocity

Fig. 5: Temperature and radial flow velocity temporal evolution at the water/air interface $(z=H)$ between $0-100 \mathrm{~s}$.

\section{B. Dynamics of the temperature and radial flow velocity}

The interest of this section is to analyze the time it takes for the flow velocity at a given distance from the laser point to attain a quasi-steady state value. This information is of importance since the flow is used to push particles lying at the water/air interface, and so it will define the particle velocity and its dynamics.

In figures $5 \mathrm{a}$ and $5 \mathrm{~b}$, it was observed that the temperature and velocity profile attain a quasi-steady state profile at the entire interface at around $100 \mathrm{~s}$. However, the regions near the laser spot seem to attain a quasi-steady state faster than the regions further away. In order to analyze this phenomenon, the temperature and flow velocity at different points at the flow interface in function of time are plotted in figures $6 \mathrm{a}$ and $6 \mathrm{~b}$. The region near the origin is affected by the heat and momentum transfer almost instantly, attaining a quasi-steady state faster than the points farther away from the origin that take longer to experience the heat and momentum propagation. 
This can be seen on figure $6 \mathrm{a}$, where at point $r=6 \mathrm{~mm}$, the temperature does not rise at all before $t=2 \mathrm{~s}$.

Also, for $r=6 \mathrm{~mm}$, figure $6 \mathrm{~b}$ shows that the radial velocity began to rise before $t=2 \mathrm{~s}$. Indeed, initially, cold water is moving towards the point, pushed by the inertia of the already moving flow in the region behind $r<6 \mathrm{~mm}$. It will be only after $2 \mathrm{~s}$ that heated water is arriving to the point.

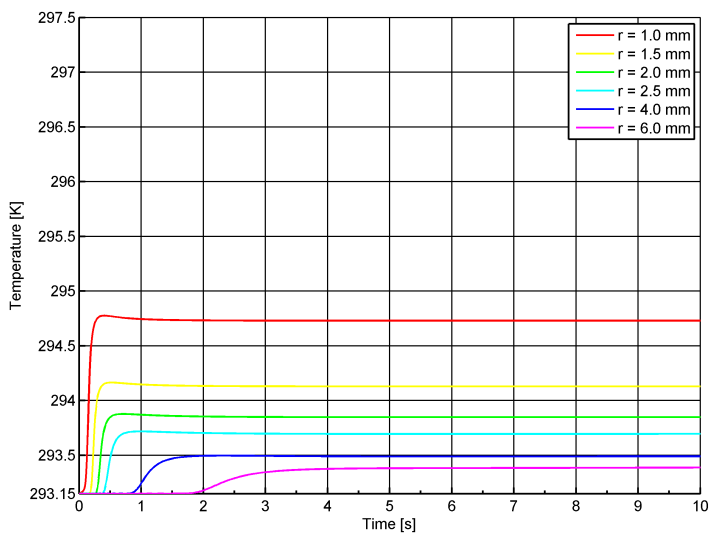

(a) Temperature at given points at the water/air interface

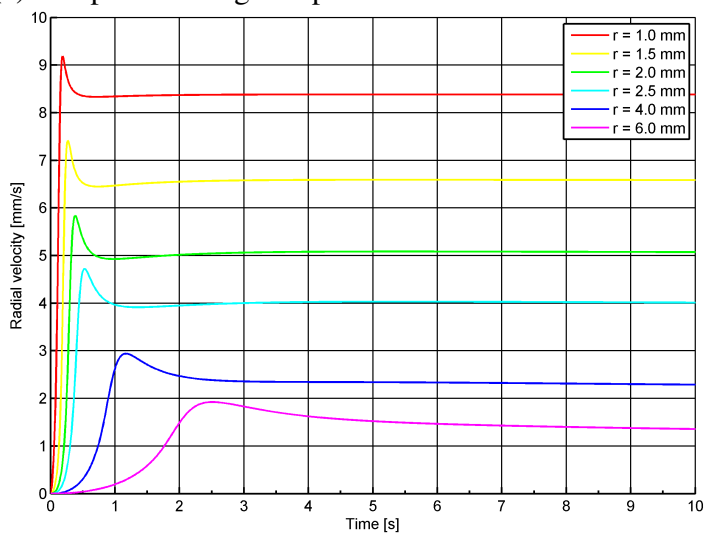

(b) Radial flow velocity at given points at the water/air interface

Fig. 6: Temperature and radial flow velocity temporal evolution at the water/air interface $(z=H)$ at the points $r=[1.0$, $1.5,2.0,2.5,4.0,6.0] \mathrm{mm}$ between $0-10 \mathrm{~s}$.

\section{Temperature and radial flow velocity at the fluid bulk}

The coupling between the temperature and the flow velocity is established at the water/air interface, from where it is propagated across the entire fluid. In this section both the temperature and radial flow velocity in the fluid bulk are shown, with special emphasis in the radial flow velocity which is the relevant parameter towards particle manipulation.

Surface plots displaying the temperature and radial velocity are shown in figures $7 \mathrm{a}$ and $7 \mathrm{~b}$, where the red arrows represent the flow velocity direction. In these plots it can be appreciated that both the temperature and the radial flow velocity are significant mostly in the upper region, near the interface. To explain this decay, it is important to remember that the surface tension stress exists only at the interface in the radial direction. So the flow velocity is the highest at the interface and has no vertical component. The only mechanism to transfer momentum to the fluid bulk is diffusion. However it was already seen that there is a large difference compared to the convective transfer mechanism. So, the momentum is transferred strongly in the radial direction but weakly in the axial direction, which explains the large decay of the radial flow velocity in the axial direction.

Another point to notice is the change of direction of the radial flow velocity. The explanation for this is the mass conservation equation (5), which establishes that the sum of the flow crossing a defined surface in the system must be 0 . This defines a convective flow cell with the top portion of the fluid having an outwards velocity, and the bottom portion having an inwards velocity to replenish the displaced fluid at the top.

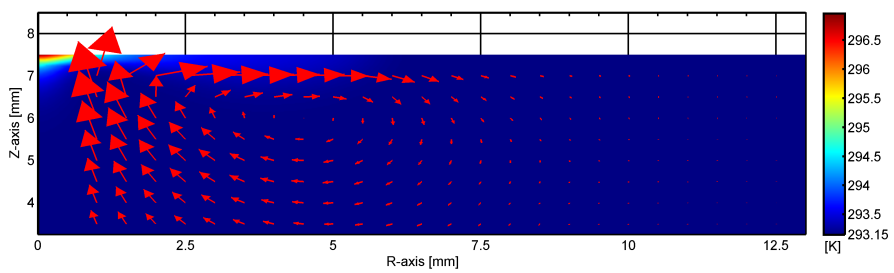

(a) Surface temperature at $\mathrm{t}=10 \mathrm{~s}$.

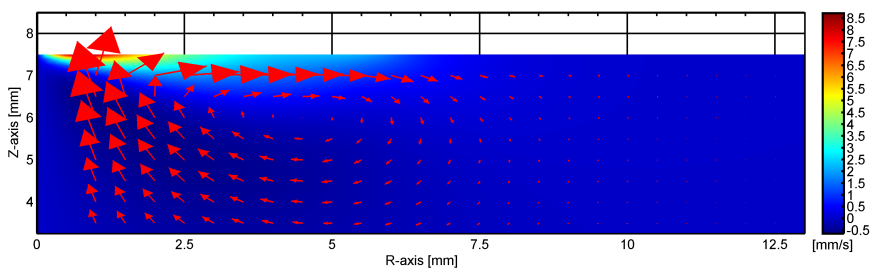

(b) Surface flow velocity at $\mathrm{t}=10 \mathrm{~s}$.

Fig. 7: Surface plots of the temperature and the flow velocity. The red arrows represent the direction of the flow on the selection points. The arrows at the water/air interface and at $r=0 \mathrm{~mm}$ were not plotted because they were much bigger in scale than the rest, and made the appreciation of the figure difficult.

In order to asses some characteristics of the convective cell, let us consider figures $8 \mathrm{a}$ and $8 \mathrm{~b}$ which present the radial flow velocity with respect to the $\mathrm{Z}$-axis coordinates at a radial distance of 1 and $2.5 \mathrm{~mm}$. Here, the conservation of mass can be appreciated, because if the flow velocity profile was to be integrated for a given time, the value of the resulting integral would be 0 . Another point to notice is that the fluid bulk takes some more time to attain a quasi-steady profile. For example, at $r=1 \mathrm{~mm}$, the radial flow velocity at the interface (fig. 6b) attains a quasi-steady state before 0.5 seconds, where the line of fluid below that point, takes around 4 seconds to stabilize. This was expected because the heat and momentum transfer in the axial direction is slower.

\section{Conclusions on the simulation results}

One of the main points to analyze in this section was the radial flow velocity which will be responsible for the particle 


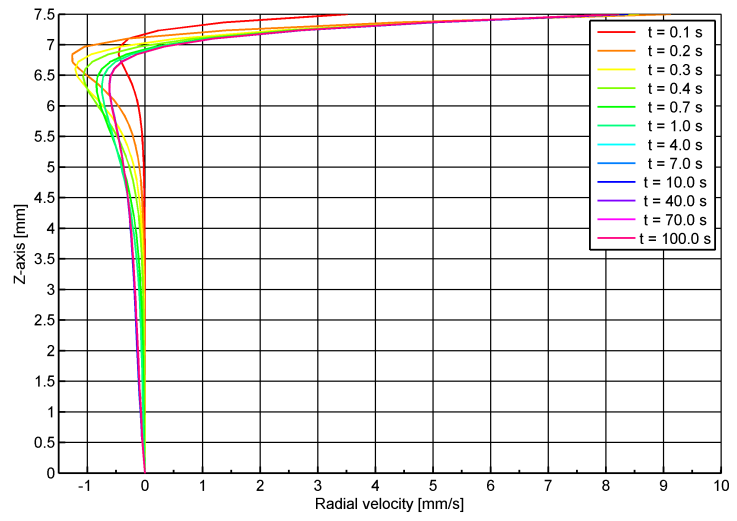

(a) $r=1 \mathrm{~mm}$

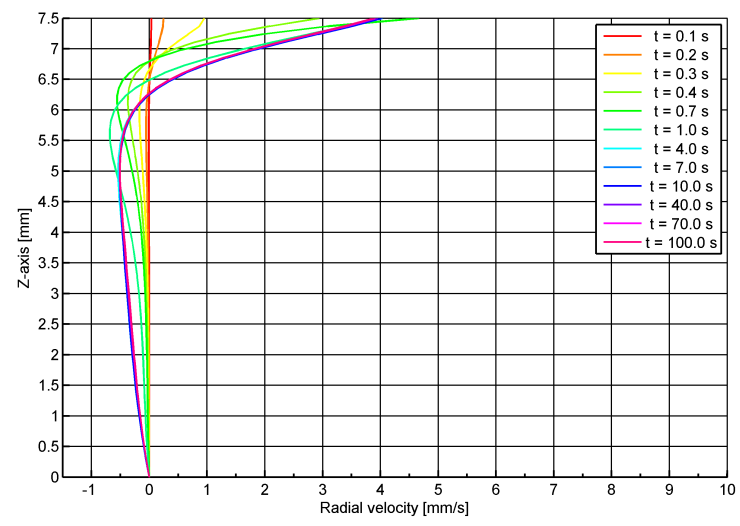

(b) $r=2.5 \mathrm{~mm}$

Fig. 8: Flow velocity variation with respect to the Z-axis in the fluid bulk at different regions.

movement. The dynamics of the radial flow velocity are of key importance because they will determine the dynamics in the particle velocity. Due to the heat and momentum propagation, the overall system attains a quasi-steady state in about 100 seconds. However, locally near the heat source $r<2 \mathrm{~mm}$, the radial flow velocity attains a quasi-steady state before $1 \mathrm{~s}$. Moreover, it is also in the region near the heat source that the radial flow velocity is the fastest, with a maximum speed at the interface in quasi-steady state of around $8.7 \mathrm{~mm} / \mathrm{s}$, which seems very promising in order to move a particle. These results would point out that the region near the laser spot is the best to manipulate particles because the dynamics of the radial flow velocity are fast and the obtained flow is the most significant. Nevertheless, the strong dependence of the radial flow velocity with the position and time needs to be considered if the position of the particle at the fluid interface is to be controlled.

\section{SETUP AND PRELIMINARY RESULTS}

In order to demonstrate that thermocapillary convection can be used for micro-object manipulation, an experimental setup has been built. With it, some experiments are performed to validate the proposed actuation method.

\section{A. Setup}

The setup consists in a laser system able to control the location of the laser and a camera to track the object position, shown in figure 9. The entire setup is mounted on a antivibration optical breadboard Newport M-IG-12-2. The laser system is composed of a continuous-wave (CW) $1455 \mathrm{~nm}$ infrared laser (Keopsys Fiber Raman Laser) which wavelength is absorbed by water. Using Beer-Lambert law, it is determined that within a water layer of approximately $900 \mu \mathrm{m}, 95 \%$ of the laser power will be absorbed (for a laser of $1455 \mathrm{~nm}$, the absorption coefficient is $3275 \mathrm{~m}^{-1}$ [39]). The laser power output was set to $200 \mathrm{~mW}$, but due to losses in the laser coupler and the different optical elements, the measured power reaching the water was of $38 \mathrm{~mW}$. The radius of the laser spot was around $0.625 \mathrm{~mm}$. Because the infrared laser is invisible to the human, it is coupled with a continuous-wave $655 \mathrm{~nm}$ red laser (KI6354 Visual Fault locator) using a laser coupler. The coupled beam is collimated using a 10x objective (Olympus Plan Achromat objective RMS10X, NA: 0.4). The collimated laser beam is directed towards a plastic container filled with distilled water, using a 2-DOF piezo-actuated tip/tilt mirror (Physik instrumente S.334-2SL) which has a tilt range of \pm $25 \mathrm{mrad}$ on each axis.

The experiments are performed using steel spherical particles with diameters of $500 \mu \mathrm{m}$ and $1000 \mu \mathrm{m}$, which are deposed at the surface of a water layer with thickness of $H \sim 7.5 \mathrm{~mm}$, contained on a square plastic container with dimensions of 115 $\times 115 \mathrm{~mm}$. The experiments are recorded using a Photonfocus camera (MV-D1024-TrackCam) which has a resolution of $512 \times 512$ pixels with $\mathrm{ROI}$ technology (region of interest) at a frame rate of $20 \mathrm{FPS}$. The camera is tilted $3.5^{\circ}$ in order to have vision of the whole working space. A $50 \mathrm{~mm}$ focal length lens together with a $10 \mathrm{~mm}$ extensor allowed to have a field of view of $49.5 \times 49.5 \mathrm{~mm}$ (one pixel represents $96.68 \mu \mathrm{m}$ ).

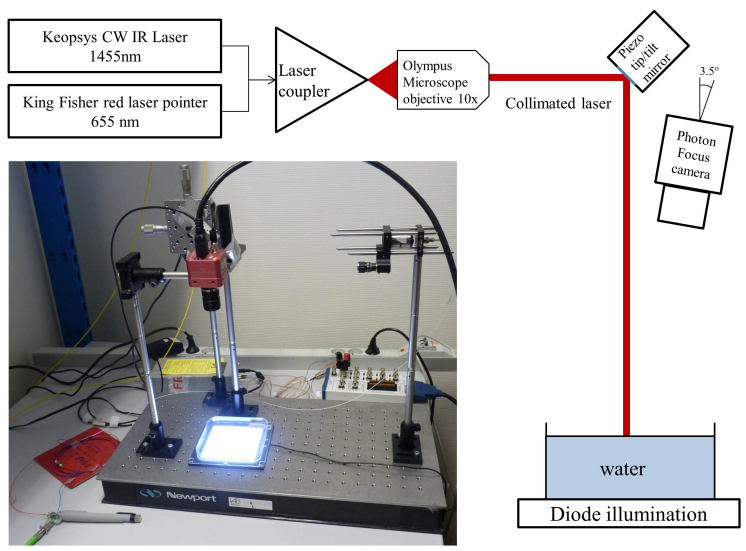

Fig. 9: Experimental setup for non-contact actuation using thermocapillary convective flows.

\section{B. Experimental validation of the proposed approach}

The objective of this proof of concept is to demonstrate that a particle can be manipulated using thermocapillary convection. The evolution of the particle position and velocity with respect to time is also analyzed. 
A spherical steel particle (AISI 304) with diameter of $500 \mu \mathrm{m}$, is deposed at the water interface. At a given instant, the laser beam is pointed next to it at a distance of $1 \mathrm{~mm}$. The laser remains still and the particle position is recorded using the camera. The experiment is performed several times on 3 different fresh water samples. Figures 10a and 10b show the results for the particle radial position and velocity, which has been obtained by taking the derivative of the position. A moving average filter with a window of 3 was applied to the position data together with the corresponding time delay compensation.

Although these results are not strictly repeatable, the same trends can be observed. The particle velocity in function of time is shown in Fig. 10b. It is important to notice that, before the laser is switched on, the particle velocity is not zero. It has a value below $1 \mathrm{~mm} / \mathrm{s}$ since it experiences fluid movements due to motion of the air above the liquid surface and slight temperature variations on the liquid surface e.g. cooling from evaporation [37]. Once the laser is pointed next to the particle at $\mathrm{t}=0 \mathrm{~s}$, the particle experiences a large acceleration after 0.1-0.3 s attaining velocities between $3-5 \mathrm{~mm} / \mathrm{s}$ (disregarding the 2 maximum and minimum values), after which the particle velocity decays as the particle moves away from the laser spot. This was expected because the radial flow velocity is significant near the laser spot where the temperature gradient is the largest, but decreases in value in the region farther away from the laser spot (Fig. 5b). However, the particle does not completely stops and the residual velocity of $1 \mathrm{~mm} / \mathrm{s}$ mentioned before persists. The attained velocity values are very promising and are at the same level of other works in the state of the art, as it will be seen in the section V-D.

In figures 10a and $10 \mathrm{~b}$, it is also displayed a rough estimation of the particle displacement and velocity obtained from COMSOL via the plot option "Particle tracing with Mass", where the mass, size and position of a spherical particle are defined in order to calculate the particle velocity using the Stokes' law. From these plots, it can be seen that most of the data fit in the region below the results given by the simulation. The ratio between the estimated particle velocity and the experimental one is in the range of 1.5-2. This points out that the Stokes' law is actually a good estimator for the resulting particle velocity, at least in the region near the heat source, despite the assumptions made (the Stokes' law does not take into account the deformation of the water/air interface due to the presence of the particle, neither the flow deviation around the particle surface).

Regarding the variability of the attained particle velocity, it is known that the water is easily contaminated by surfactants [40]. Because of the large value of surface tension of water, surfactants may dramatically change the stability properties of the convective flow [41]. So the more time passes, more surfactants may come to the air/water interface, changing the resulting flow velocity. This variability has a repercussion in the particle displacement, shown in Fig. 10a, where the

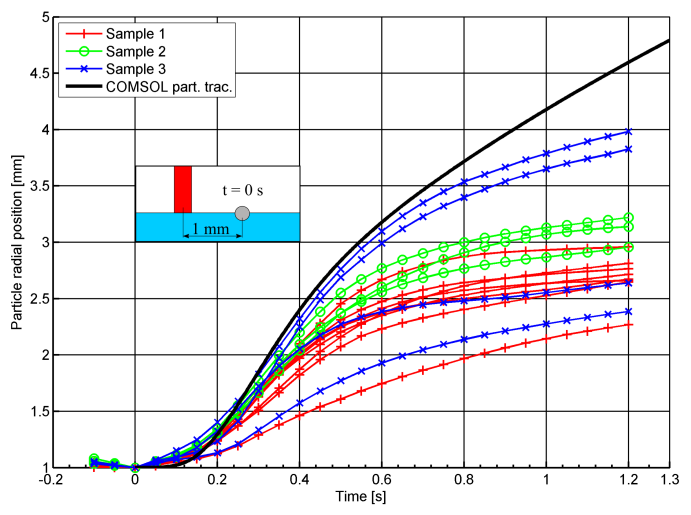

(a) Particle position

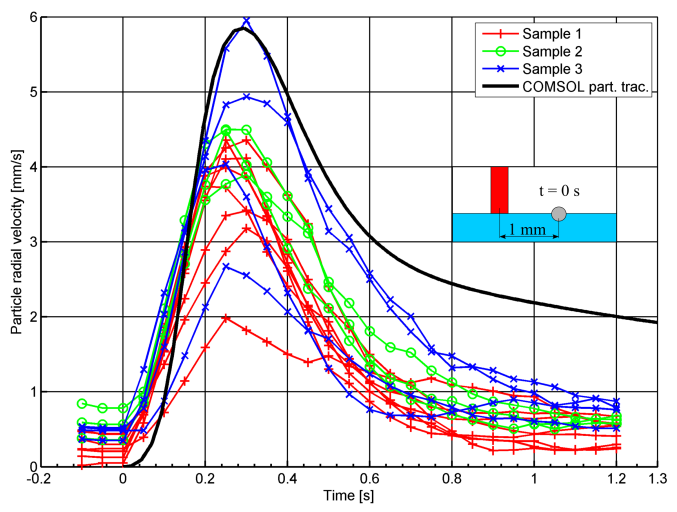

(b) Particle velocity

Fig. 10: Computed particle position and velocity in function of time, when the laser beam is pointed $1 \mathrm{~mm}$ away from the $500 \mu \mathrm{m}$ spherical steel particle at time $=0 \mathrm{~s}$. A moving average filter with a window of 3 was applied to the particle position together with the corresponding delay compensation. Several trials were performed using 3 different water samples. These trials were performed shortly after the distilled water was deposed on the container. The experimental results are compared to the simulation results obtained from COMSOL using the module "Particle tracing with mass". (Video available as supplementary material).

particles displaced different distances for the same input. Disregarding the two top and bottom extreme cases, the variation is of around $0.7 \mathrm{~mm}$, meaning a variation of $23.3 \%$ with respect to the average traveled distance of $3 \mathrm{~mm}$.

\section{First manual trajectory tracking}

During the experimental tests performed in section V-B, it was appreciated that the particle moved in the direction of the line connecting the centers of the particle and the laser spot. Having this idea in mind, a trajectory tracking test was performed on which it is shown that the particle can be moved along an "8" shape by manually controlling the laser position. The result is shown in Fig. 11. Even though some errors can be seen in the trajectory tracking, it shows that the direction of movement can be controlled. This opens the possibility to control the position of the particle at the interface. 


\section{Discussion}

Table III shows a rough comparison between the proposed method and the techniques from the state of the art based on surface tension gradients, presented in section II-C.

TABLE III: Micro manipulators using thermal induced surface tension gradients

\begin{tabular}{|c|c|c|c|}
\hline Reference & Method & Vel. max $\mathrm{mm} / \mathrm{s}$ & Net Force \\
\hline Vela [23] & Mar. Conv & $V_{\text {part }} 5.5$ & $40 \mathrm{pN}$ \\
\hline Hendarto et al. [30] & Thcap. Conv. & - & $0.1 \mathrm{nN}$ \\
\hline Hendarto et al. [31] & Mar. Conv. & $V_{\text {flow }} 18.7$ & - \\
\hline Ishii et al. [32] & Thcap. Conv. & - & $\begin{array}{c}F_{\text {push }} 1.6 \mathrm{nN} \\
F_{\text {pull }} 0.6 \mathrm{nN}\end{array}$ \\
\hline Hu et al. [33] & Thcap. Conv. & $V_{\text {part push } 0.2}$ & - \\
& & $V_{\text {part pull }} 0.1$ & \\
\hline On device & Thcap. Conv. & $V_{\text {part. }} 4$ & $\sim 10 \mathrm{nN}$ \\
\hline
\end{tabular}

In table III, "Thcap. Conv." stands for thermocapillary convection and "Mar. Conv." stands for Marangoni convection. The velocity was obtained from the first derivative of the mean filtered particle position data. The force was obtained from the product of the computed acceleration, which was obtained from second derivative of the mean filtered particle position data, and the mass.

The attained particle velocity of $4 \mathrm{~mm} / \mathrm{s}$ using the current system is one of the fastest only below the results presented by Vela [23]. However, the force exerted on the particle is more than 200 times than the force reported by Vela [23], and more than 5 times the force reported by Ishii et al. [32]. The main reason for this large difference is that, in the current proposal, the particle is manipulated at the fluid/gas interface where the surface tension stress is the strongest, different from the other authors who manipulate the particle in the fluid bulk. In addition, thermocapillary convection is used, on which the interface is directly heated by the laser. This reduces the power required to heat the interface compared to the authors who used Marangoni convection where the heat comes from the bottom or authors who used a light absorbent substrate to generate heat in the fluid. These results are promising for applications where only planar displacements are needed. Such a need has been expressed at microscale in several works ([42], [43], [44], [45], [46]).

However, there are some issues to be addressed in the future. One of them is that the particle is never still, but it is constantly moving with a velocity below $1 \mathrm{~mm} / \mathrm{s}$. Because of this, constant actuation would be required in order to keep the particle on a given position. Another problem is the surface contamination, which is constantly changing the surface tension properties which has a significant impact in the thermocapillary flow, and consequently, in the particle velocity. Closed-loop control will be considered in the future to address these issues. It is important to mention that in order to manipulate a particle using the current proposal, this should float at the water/air interface. Therefore the weight, and contact angle of the particle must be taken into account.

\section{CONCLUSION}

This paper proposes to exploit thermocapillary convection in micro-object manipulation applications. Using this principle, fluid flow is generated using a laser beam that heats the water/air interface generating a surface tension stress that drives the fluid motion. The experimental results show that particles can be displaced at the water/air interface using thermocapillary convection. Manually controlling the position of the laser, the movement of a $1000 \mu \mathrm{m}$ diameter steel microsphere has been controlled along a given trajectory. The mean maximal measured velocity is $4 \mathrm{~mm} / \mathrm{s}$, which is in top performance compared to other principles based on surface tension gradients. Although the experiments present some variations between different trials, the trends of the different curves clearly show that the particle can be manipulated via the thermocapillary convective flow. Vision-based closed-loop control will enable, in future works, to improve both the repeatability and the velocity of the micromanipulations.

\section{ACKNOLEDGEMENTS}

This research has been funded by the Interuniversity Attraction Poles Programme (IAP 7/38 MicroMAST) initiated by the Belgian Science Policy Office, by the Labex ACTION project (contract "ANR-11-LABX-01-01"), by the French Agence Nationale de la Recherche, through the LEMA project (contract "ANR 12 BS03 007 01"), by the PHC Tournesol (contract "FR 2015 project 34237PC") and by the Région FrancheComté. This work has also been carried out in relation with the COST Action MP1106 on Smart and Green Interfaces. The authors would like to thank Prof. Pascal Kockaert for his valuable advices in laser optics, and Prof. Pierre Colinet for his valuable advices regarding the surface tension phenomena and thermocapillary convection.

\section{REFERENCES}

[1] E. B. Steager, M. S. Sakar, C. Magee, M. Kennedy, A. Cowley, and V. Kumar, "Automated biomanipulation of single cells using magnetic microrobots," en, The International Journal of Robotics Research, vol. 32, no. 3, pp. 346-359, Mar. 2013.

[2] S. Bouchebout, A. Bolopion, M. Gauthier, and S. Regnier, "Position control of a ferromagnetic micro-particle in a dry environment," in IEEE/ASME International Conference on Advanced Intelligent Mechatronics, 2014, pp. 1-6.

[3] M. Mehrtash, X. Zhang, and M. Khamesee, "Bilateral magnetic micromanipulation using off-board force sensor," Mechatronics, IEEE/ASME Transactions on, vol. 20, no. 6, pp. 3223-3231, 2015.

[4] H. Uvet, L. Feng, S. Ohashi, M. Hagiwara, T. Kawahara, Y. Yamanishi, and F. Arai, "On-chip single particle loading and dispensing," in 2011 IEEE International Conference on Robotics and Automation (ICRA), May 2011, pp. $3151-3156$.

[5] J. A. Piepmeier, S. Firebaugh, and C. S. Olsen, "Uncalibrated visual servo control of magnetically actuated microrobots in a fluid environment," Micromachines, vol. 5, no. 4, pp. 797-813, Sep. 2014.

[6] M. P Kummer, J. J Abbott, B. E Kratochvil, R. Borer, A. Sengul, and B. J Nelson, "OctoMag: An Electromagnetic System for 5-DOF Wireless Micromanipulation," English, IEEE Transactions on Robotics, vol. 26, no. 6, pp. 1006-1017, Dec. 2010.

[7] J. Keuning, J. de Vries, L. Abelmann, and S. Misra, "ImageBased Magnetic Control of Paramagnetic Microparticles in Water," in IEEE/RSJ International Conference on Intelligent Robots and Systems, 2011, pp. 421-426.

[8] E. Diller, J. Giltinan, and M. Sitti, "Independent control of multiple magnetic microrobots in three dimensions," en, The International Journal of Robotics Research, vol. 32, no. 5, pp. 614-631, Apr. 2013. 


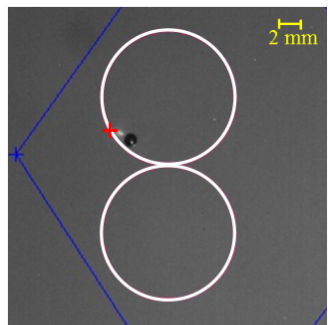

a) $\mathrm{t}=0 \mathrm{~s}$

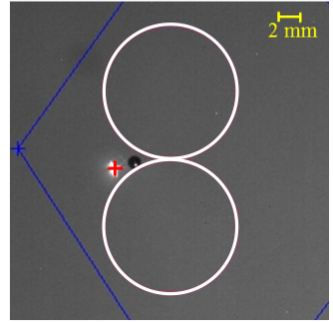

f) $\mathrm{t}=30.25 \mathrm{~s}$

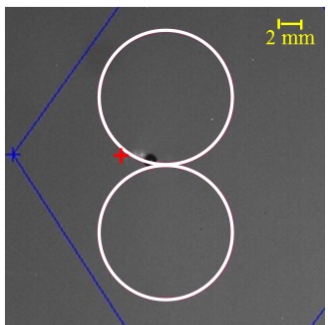

b) $\mathrm{t}=3 \mathrm{~s}$

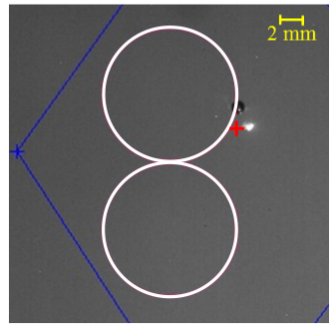

g) $t=37.5 \mathrm{~s}$

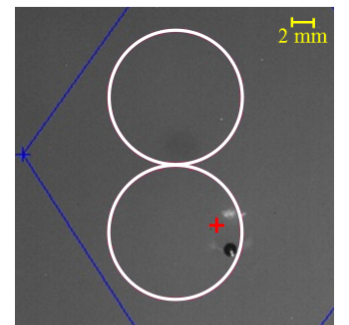

c) $\mathrm{t}=12 \mathrm{~s}$

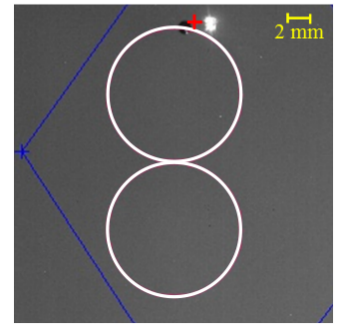

h) $\mathrm{t}=48 \mathrm{~s}$

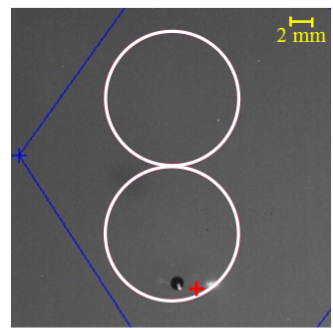

d) $\mathrm{t}=18.5 \mathrm{~s}$

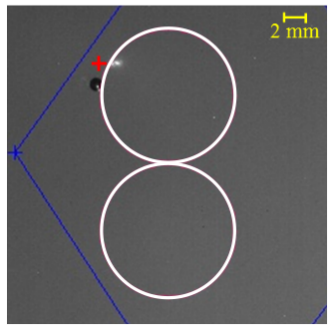

i) $\mathrm{t}=54.5 \mathrm{~s}$

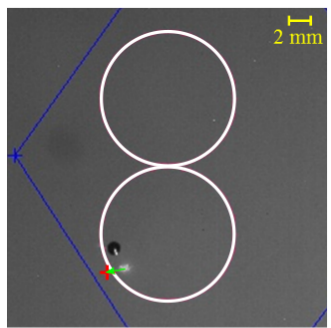

e) $t=24.5 \mathrm{~s}$

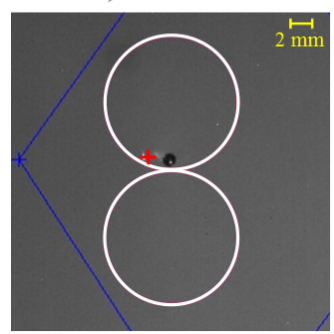

j) $\mathrm{t}=64.5 \mathrm{~s}$

Fig. 11: Example of a $1 \mathrm{~mm}$ diameter spherical zirconium particle (black circle) following a "8" shape trajectory (shown as the white line) at different time instants. The red cross is the position of the laser. The area inside the blue lines defines the space on which the laser can be pointed. The original pictures were cropped and zoomed to show the region of interest. (Video available as supplementary material).

[9] H. Pohl, Dielectrophoresis. Cambridge University press, 1978.

[10] J. Zemanek, J. Drs, and Z. Hurak, "Dielectrophoretic actuation strategy for micromanipulation along complex trajectories," in Advanced Intelligent Mechatronics (AIM), 2014 IEEE/ASME International Conference on, 2014, pp. 19-25.

[11] S.-W. Nam, S. H. Kim, J.-K. Park, and S. Park, "Dielectrophoresis in a slanted microchannel for separation of microparticles and bacteria," Journal of nanoscience and nanotechnology, vol. 13, no. 12, pp. 79937997, 2013.

[12] J. Voldman, "Electrical forces for microscale cell manipulation," eng, Annual Review of Biomedical Engineering, vol. 8, pp. 425-454, 2006.

[13] M. Kharboutly and M. Gauthier, "High speed closed loop control of a dielectrophoresis-based system," in International Conference on Robotics and Automation, 2013, pp. 1446-1451.

[14] A. Ashkin, J. Dziedzic, J. Bjorkholm, and S. Chu, "Observation of a single-beam gradient force optical trap for dielectric particles," Optics letters, vol. 11, no. 5, pp. 288-290, 1986.

[15] K. Dholakia, G. Spalding, and M. MacDonald, "Optical tweezers: the next generation," Physics world, vol. 15, no. 10, pp. 31-36, 2002.

[16] K. Onda and F. Arai, "Parallel teleoperation of holographic optical tweezers using multi-touch user interface," in IEEE International Conference on Robotics and Automation, May 2012, pp. 1069 -1074.

[17] X. Li and C. C. Cheah, "Robotic cell manipulation using optical tweezers with unknown trapping stiffness and limited fov," Mechatronics, IEEE/ASME Transactions on, vol. 20, no. 4, pp. 1624-1632, 2015.

[18] M. Xie, Y. Wang, G. Feng, and D. Sun, "Automated pairing manipulation of biological cells with a robot-tweezers manipulation system," Mechatronics, IEEE/ASME Transactions on, vol. 20, no. 5, pp. 2242-2251, 2015.

[19] M. A. Burns, C. H. Mastrangelo, T. S. Sammarco, F. P. Man, J. R. Webster, B. N. Johnsons, B Foerster, D Jones, Y Fields, A. R. Kaiser, and D. T Burke, "Microfabricated structures for integrated dna analysis," in Proceedings of the National Academy of Sciences of the United States of America, 93(11), vol. 93, 1996, pp. 5556-5561.

[20] J. O. Kessler, "Hydrodynamic focusing of motile algal cells," Nature, vol. 313, pp. 218-220, 1985.

[21] G.-B. Lee, B.-H. Hwei, and G.-R. Huang, "Micromachined pre-focused $\mathrm{m} \times \mathrm{n}$ flow switches for continuous multi-sample injection," Journal of Micromechanics and Microengineering, vol. 11, no. 6, p. 654, 2001.

[22] N. Sundararajan, M. Pio, L. P. Lee, and A. Berlin, "Three-dimensional hydrodynamic focusing in polydimethylsiloxane (pdms) microchannels," Microelectromechanical Systems, Journal of, vol. 13, no. 4, pp. 559-567, 2004.
[23] E. Vela, "Non-contact microscale manipulation using laser-induced convection flows," PhD thesis, Universite Pierre et Marie Currie, 2010.

[24] P.-G. de Gennes, F. Brochard-Wyart, and D. Quéré, Capillarity and Wetting Phenomena: Drops, Bubbles, Pearls, Waves, Springer, Ed. Springer, 2004.

[25] P. Agarwal and S. Sinha, Elements of Physics XI, R. K. Rastogi, Ed. Rastogi Publications, 2009.

[26] W. Ramsay and J. Shields, "Lxxxi.-the molecular complexity of liquids," J. Chem. Soc., Trans., vol. 63, pp. 1089-1109, 01893.

[27] E. Guyon, J.-P. Jaeger, and L. Petit, Physical Hydrodynamics, 2nd. Oxford University Press, 2001.

[28] M. F. Schatz and G. P. Neitzel, "Experiments on thermocapillary instabilities," Annual Review of Fluid Mechanics, vol. 33, no. 1, pp. 93-127, 2001.

[29] E. Vela, C. Pacoret, S. Bouchigny, S. Regnier, K. Rink, and A. Bergander, "Non-contact mesoscale manipulation using laser induced convection flows," in Intelligent Robots and Systems (IROS), 2008, pp. 913-918.

[30] E. Hendarto and Y. B. Gianchandani, "A non-contact method for spatially localized sedimentation of particles from liquid suspensions using marangoni forces," Journal of Micromechanics and Microengineering, vol. 21, no. 11, p. $115028,2011$.

[31] _ _ "Size sorting of floating spheres based on marangoni forces in evaporating droplets," Journal of Micromechanics and Microengineering, vol. 23, no. 7, p. $075016,2013$.

[32] K. Ishii, W. Hu, and A. Ohta, "Cooperative micromanipulation using optically controlled bubble microrobots," in Robotics and Automation (ICRA), 2012 IEEE International Conference on, 2012, pp. 3443-3448.

[33] W. Hu, Q. Fan, and A. T. Ohta, "An opto-thermocapillary cell micromanipulator," Lab Chip, vol. 13, pp. 2285-2291, 122013.

[34] H. Chraïbi and J.-P. Delville, “Thermocapillary flows and interface deformations produced by localized laser heating in confined environment," Physics of Fluids (1994-present), vol. 24, no. 3, 2012.

[35] M. Robert de Saint Vincent and J.-P. Delville, "Thermocapillary migration in small-scale temperature gradients: application to optofluidic drop dispensing," Phys. Rev. E, vol. 85, p. 026310, 22012.

[36] S. P. Karlov, D. A. Kazenin, B. I. Myznikova, and I. I. Wertgeim, "Experimental and numerical study of the marangoni convection due to localized laser heating," Journal of Non Equilibrium Thermodynamics, vol. 30, pp. 283-304, 2005.

[37] J. P. Longtin, K. Hijikata, and K. Ogawa, "Laser-induced surfacetension-driven flows in liquids," International Journal of Heat and Mass Transfer, vol. 42, no. 1, pp. 85 -93, 1999. 
[38] A. Bejan, "External natural convection," in Convection Heat Transfer, I. John Wiley \& Sons, Ed. John Wiley \& Sons, Inc., 2013, ch. 4, pp. $168-232$.

[39] J. Xu, K. W. Plaxco, and S. J. Allen, "Absorption spectra of liquid water and aqueous buffers between 0.3 and 3.72thz," The Journal of Chemical Physics, vol. 124, no. 3, 036101, pp. -, 2006.

[40] E. Hendarto, "Applications of marangoni forces in actuating solid phase objects," PhD thesis, University of Michigan, 2013.

[41] J. Berg and A. Acrivos, "The effect of surface active agents on convection cells induced by surface tension," Chemical Engineering Science, vol. 20, no. 8, pp. $737-745,1965$.

[42] J. M. K. Ng, M. J. Fuerstman, B. A. Grzybowski, H. A. Stone, and G. M. Whitesides, "Self-assembly of gears at a fluid/air interface," Journal of the American Chemical Society, vol. 125, no. 26, pp. 7948-7958, 2003, PMID: 12823016.

[43] A. Jesacher, S. Fürhapter, C. Maurer, S. Bernet, and M. Ritsch-Marte, "Holographic optical tweezers for object manipulations at an air-liquid surface," Opt. Express, vol. 14, no. 13, pp. 6342-6352, 2006.

[44] S. Gupta, N. Singh, M. Sastry, R. Kakkar, and R. Pasricha, "Controlling the assembly of hydrophobized gold nanoparticles at the air-water interface by varying the interfacial tension," Thin Solid Films, vol. 519 no. 3, pp. $1072-1077,2010$, Biomolecular Electronics and Organic Nanotechnology for Environmental Preservation.

[45] P. Rogers, I. Gralinski, C. Galtry, and A. Neild, "Selective particle and cell clustering at air-liquid interfaces within ultrasonic microfluidic systems," English, Microfluidics and Nanofluidics, vol. 14, no. 3-4, pp. 469-477, 2013.

[46] M. Dkhil, M. Kharboutly, A. Bolopion, S. Regnier, and M. Gauthier, "Closed-loop control of a magnetic particle at the air-liquid interface," Automation Science and Engineering, IEEE Transactions on, vol. PP, no. 99, pp. 1-13, 2015. 